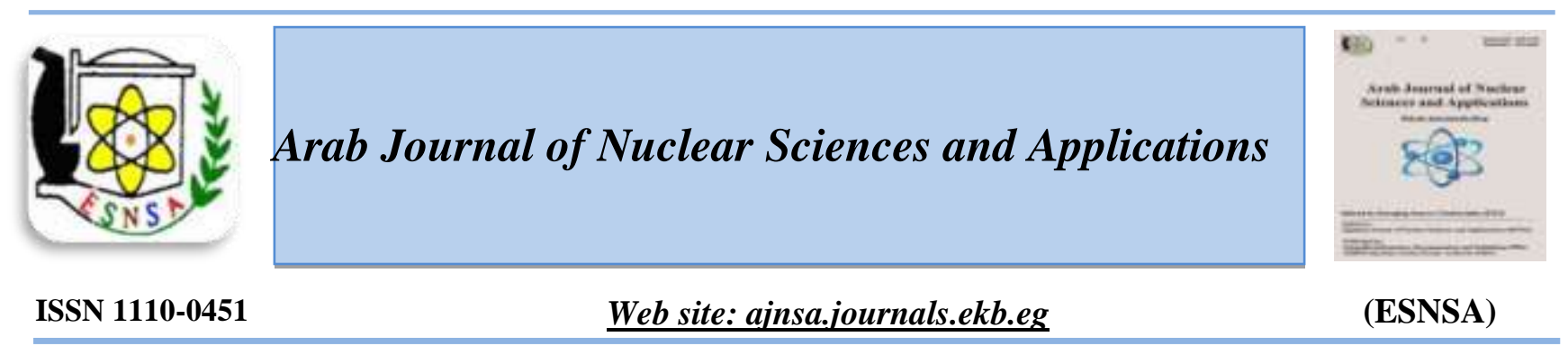

\title{
Quantification of Various Factors Influencing Repeatability and Reproducibility of TLD-600 Detector
}

\author{
A.I. Abd El-Hafez ${ }^{a}$, A.O. El-Sheikh ${ }^{b, c}$, M.M. Ahmed ${ }^{c}$, Y.A. Abdel-Razek ${ }^{d}$ and M.S. El-Nagdy ${ }^{c}$ \\ ${ }^{a}$ Ionizing Radiation Metrology Laboratory, National Institute for Standards (NIS) Giza, Egypt \\ ${ }^{b}$ Egyptian Customs Authority, Central Administration for Technology of Compliance, Alexandria, Egypt \\ ${ }^{c}$ Physics Department, Faculty of Science, Helwan University, Cairo, Egypt \\ ${ }^{d}$ Nuclear Materials Authority, Cairo, Egypt
}

\begin{abstract}
Received $23^{\text {th }}$ Mar. 2019 Various factors affecting the random and systematic error in the repeatability and reproducibility of Accepted $17^{\text {th }}$ Sep. 2019 thermoluminescent ${ }^{6} \mathrm{LiF}: \mathrm{Mg}$,Ti dosimeters were investigated. These characteristics were examined by irradiating them at different doses in the range $3.8 \mu \mathrm{G}$ to $433.94 \mathrm{mGy}$ from a Cs-137 gamma source. Values of the element correction factors (ECFs) were obtained by exposing the chips to a dose of 433.93 mGy five times. This investigation denotes that more correction will be obtained by calculating the error due to non-subtracting zero dose reading from the delivered dose, which decreases the coefficient of variation as a percentage of the mean from the range of $2.4 \%-18.6 \%$ to only $0.7 \%-16.9 \%$. Using the individual sensitivity factor and repeating five calibrations decreases the coefficient of variation as a percentage of the mean to only $0.54 \%$. The values of the Variability Index for System (SVI), Reader (RVI) and Detector (DVI) are $1.46 \%, 1.40 \%$ and $0.34 \%$ respectively. Also, it is clear from the results that the best repeatability and reproducibility obtained by using the element correction factor was associated with each dosimeter rather than carefully selecting the individual dosimeters. Finally, from the results, it can be noted that at low doses, the value of relative coefficient of variation becomes very large, while at high doses, it becomes basically constant and of the order of a few percentage.
\end{abstract}

Keywords: Thermoluminescence dosimetry, TLD-600, Repeatability, Reproducibility, Variability index

\section{Introduction}

The repeatability of measurements with thermoluminescence dosimeters is one of the most important issues that ought to be taken into account. Moreover, the reproducibility in TL dosimetry should be improved by decreasing the errors associated with the maximum amount as low as possible [1]. The subsequent factors are responsible for determining the dosimetry properties of a TLD system: thermoluminescence reader (PM dark and read out profile), thermoluminescence material (batch quality and batch history) and annealing proceeding calibration irradiation system. Furthermore, the background reading (zero dose) of TLDs plays a very

important role in all accurate dosimetry proceedings in order to correct additive doses appearing from alternative supplies as compared to irradiation processes [2-4].

For performing exact dosimetry, a good knowledge of optimum dosimetry procedures should be available. Energy, rate of pre-irradiation, rate of post- irradiation, annealing, optimum time, time-temperature profile (TTPs) for TLD read out and force field homogeneity are very serious issues in improvement of TLD responses[5-10]. Plato and Miklos explained in detail how to create and calibrate a TLD system for radiation observation [11].They supported the estimation of ECFs for

Corresponding author: abdelhamidalsheikh@yahoo.com

DOI: 10.21608 /ajnsa.2019.13313.1219

(C) Scientific Information, Documentation and Publishing Office (SIDPO)-EAEA 
personal dosimetry, and they gave proceedings for how to produce element coefficient factors for thermoluminescence dosimeters system where the reproducibility of a set of TLD dosimeters can be improved by these proceedings, also the relative coefficient of variation will be decreased.

The main benefit of using individual coefficient factors through thermoluminescence dosimetry is that deviations that were represented by these element coefficient factors are deviations within the TL elements and not deviations caused by stability problems with the TLD reader. Moreover, the results of reproducibility are also dependent on the irradiation dose.

Burkhardt and Piesh [12] studied the reproducibility of TLD systems. They calculated the relative coefficient of variation of the thermoluminescence readings as a function of the dose received by 10 dosimeters, and presented it graphically as a function of the dose. They were able to present the results by a two-parameter fit of the form:

$S(D)=\left\lceil\frac{A^{2}}{D^{2}}+B^{2}\right\rceil^{1 / 2}$

Where $A$ is the absolute relative coefficient of variation at very low doses in dose units and $B$ is the relative coefficient of variation at a high dose in reader units.

A slight difference in the mathematical expression for the dependence of relative coefficient of variation on the dose received by the detectors has been developed by Zarand and Polgar [13-14]. They found that in some experimental situations, the relative coefficient of variation of the data could be presented more accurately by the expression:

$\mathrm{S}(\mathrm{D})=\left\lceil\frac{\mathrm{A}^{2}}{\mathrm{D}^{2}}+\frac{1}{\mathrm{kD}}+\mathrm{B}^{2}\right\rceil^{1 / 2}$

An additional term $1 / \mathrm{kD}$ appears in this formula, where $\mathrm{D}$ refers to the absorbed dose as in the prior formula while $\mathrm{k}$ is a constant showing the conversions factor of photoelectron-to-dose.

This study aims at examining the repeatability and reproducibility of the response of $6 \mathrm{LiF}: \mathrm{Mg}, \mathrm{Ti}$ thermoluminescent detector and investigating the variability of the whole thermoluminescence system (SVI) and individually to investigate the variability of the thermoluminescence reader (RVI) and the variability of the thermoluminescence dosimeters (DVI).

\section{Material and methods}

Ten dosimeters of ${ }^{6} \mathrm{LiF}: \mathrm{Mg}, \mathrm{Ti}$ (TLD-600) with dimensions $3.2 \times 3.2 \times 0.89 \mathrm{~mm}^{3}$ were used in this experiment. When not being read or irradiated, the TLDs were stored in dark at room temperature.

Glow curves were obtained using TL-readout system of the type Harshaw 4500, manufactured by Harshaw chemical company in Ohio, USA.

The dosimeters were annealed in an oven at $400{ }^{\circ} \mathrm{C}$ for 1 hour, and then were cooled down in the opened oven till reaching a temperature of $\sim 60^{\circ} \mathrm{C}$. The oven was then closed and heated up to $100^{\circ} \mathrm{C}$ for 2 hours and the dosimeters were then cooled down in air. The dosimeters had the pre- and postirradiation readings at heating rate of $3{ }^{\circ} \mathrm{C} / \mathrm{sec}$ over the temperature readout range of $100-400{ }^{\circ} \mathrm{C}$.

Irradiation was performed at room temperature using ${ }^{137} \mathrm{Cs}$ gamma source model GB-150 with an original activity of a thousand $\mathrm{Ci}$ manufactured by the Atomic Energy of Canada Limited and designed for use in a shielded room. For irradiation, the TLDs were irradiated at doses of $3.8,19.0,45.4,75.7,189.2$, and $435.1 \mu \mathrm{Gy}$, and $3.894,19.470,38.939,43.394,86.788,216.969$ and $433.94 \mathrm{mGy}$ from the gamma source with the help of different thickness of lead sheets.

TL dosimeters were annealed, and kept in the dark. All dosimeters were grouped together in one unit composed_of 10 dosimeters of TLD-600 detector. The experimental proceedings consisted of two basic steps. First: the readings of zero doses were carried out for all chips. Second: the ten chips were grouped together and irradiated with a welldefined radiation dose from ${ }^{137} \mathrm{Cs}$ gamma supply. These procedures were repeated five times in identical manner.

To omit the impact of methodical error in TLD responses, the repeatability of element correction factor (ECF) was examined, which was a relative dose.

The repeatability of TLD-600 response was calculated as a function of the absorbed dose using the following formula [15]:

$$
R=\frac{\sigma}{\bar{M}} \times 100 \% \leq 7.5 \%
$$

Where $\mathrm{M}$ is the area under the glow curve from $100-400{ }^{\circ} \mathrm{C}$ and $\bar{M}$ is the average of all readings all over the five cycles and $\sigma$ is the standard deviation.

The coefficient of variation can be defined as the system variability index (SVI). It is the mean value of the coefficient percentage of variation of every 
TL detector and this amount provides a reproducibility of the whole system, reader variability index (RVI), which is the percentage of coefficients of mean value of the variation of every cycle of readings and this amount provides a long term reader reproducibility and detector variability index (DVI) and this amount provides a reproducibility of the TL detectors and it is defined as:

$$
D V I=\sqrt{(S V I)^{2}-(R V I)^{2}}
$$

The variation of the coefficient of variation $S(D)$ of the TL readings for different doses $\mathrm{D}$ can express the reproducibility of a TLD system. The formula (1) often expresses this variation.

\section{Results and discussion}

A visual checking of the TLDs samples showed that there were no chips suffering a visible physical harm. All chips had no missing corners, no heavy scratches and no stains. Therefore, during this study, the effect of the physical damage on the accuracy of the TLD system was not discussed.

There were no thermoluminescence dosimeters having multiple measurements, so far, the sample which have been a result of coefficients unconcerned to the precise TLD. For instance, high values might be a result of the burning of dust on the planchet. The low values might be a result of unsuitable placement of the thermoluminescence dosimeter on the planchet, inflicting the thermoluminescence dosimeter not be heated the maximum amount to supply a full signal.

Element correction factors

Figure (1) shows the percentage of sample coefficient of variation for each TLD dosimeter. The data was presented in this Figure without subtracting zero dose reading. This Fig. shows that the relative coefficient of variation $(\mathrm{CV} \%)$ reached a value of $3.93 \%$ for TLD-600 dosimeters which is less than $7.5 \%$ for all TLDs as in formula 3. This value is not accepted for primary standard dosimetry lab (needs to be $\leq 1.5 \%$ ) or secondary standard dosimetry lab (needs to be $\leq 3.5 \%$ ). To reduce the coefficient of variation the zero doses from the data represented in Fig. (1) should be substracted. The result is shown in Fig. (2) which presents the percentage variation for the element correction factor (ECF) of each TLD after subtracting zero doses from the data.

Figure (2) shows that the maximum value of the relative coefficient of variation $(\mathrm{CV} \%)$ of element correction factor (ECF) is $2.37 \%$ for TLD-600 dosimeters which is less than 3.932 as shown in Fig. (1).

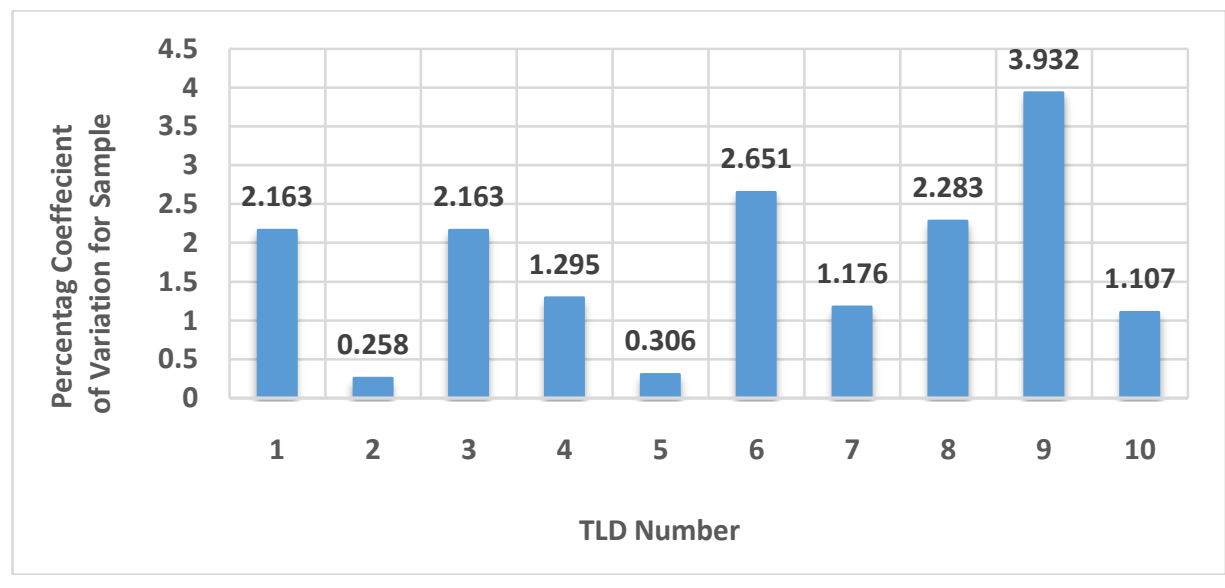

Fig. (1): The percentage sample coefficient of variation (CV\%) for each TL dosimeter

To omit the impact of systematic error in TLD responses, the repeatability for each chip from the ten chips was examined to obtain the element correction factor. Once, a correction of dosimeters readings was done, these data indicates a relative decrease in the standard deviation of further irradiations. From the obtained result, it was found that the deviation of data decreased after utilizing the element correction factor of $0.54 \%$ as shown in Table (1). Table (1) presents the corrected readings of ten thermoluminescence dosimeters exposed to identical dose of 433.9 mGy from Cs-137 gamma source. This proceeding has been performed over identical five cycles. A statistical method was used 
to calculate the repeatability and reproducibility of TLD-600 dosimeters system. The average, the standard deviation and the covariance for each corrected reading over the five cycles were calculated.

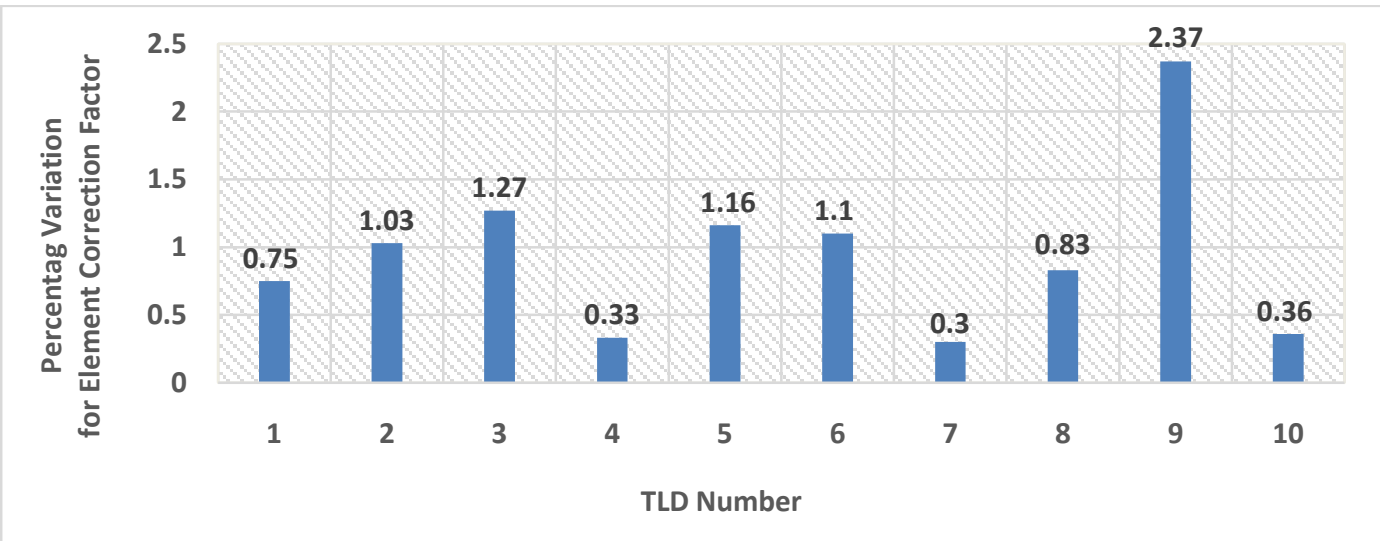

Fig. (2): The percentage variation for the element correction factor (ECF) of each TLD

Table (1): Data table for repeatability and reproducibility of corrected TLD-600 measurements

\begin{tabular}{l|l|l|l|l|l|l|l|l}
\hline \multirow{2}{*}{$\begin{array}{l}\text { TLD-600 } \\
\text { dosimeter } \\
\text { No. }\end{array}$} & \multicolumn{7}{c}{ TL corrected count reader unit } \\
\cline { 2 - 8 } & $\mathbf{1}^{\text {st }}$ & $\mathbf{2}^{\text {nd }}$ & $\mathbf{3}^{\text {rd }}$ & $\mathbf{4}^{\text {th }}$ & $\mathbf{5}^{\text {th }}$ & $\begin{array}{c}\text { Mean value } \\
\text { of the five } \\
\text { readings }\end{array}$ & STDEV & CV\% \\
\hline 1 & 14139 & 14329 & 14715 & 14778 & 14857 & 14566 & 312 & 2.1 \\
2 & 14534 & 14540 & 14575 & 14597 & 14593 & 14564 & 29 & 0.2 \\
3 & 14567 & 14560 & 14561 & 14561 & 14565 & 14563 & 3 & 0.021 \\
4 & 14316 & 14433 & 14606 & 14710 & 14746 & 14562 & 184 & 1.3 \\
5 & 14543 & 14532 & 14579 & 14574 & 14581 & 14563 & 22 & 0.2 \\
6 & 14029 & 14334 & 14689 & 14877 & 14902 & 14566 & 376 & 2.6 \\
7 & 14312 & 14477 & 14658 & 14674 & 14683 & 14563 & 163 & 1.1 \\
8 & 14013 & 14605 & 14708 & 14730 & 14763 & 14565 & 313 & 2.2 \\
9 & 13603 & 14538 & 14810 & 14932 & 14987 & 14575 & 570 & 3.9 \\
10 & 14312 & 14532 & 14632 & 14667 & 14666 & 14562 & 150 & 1.0 \\
Average & 14237 & 14488 & 14653 & 14710 & 14734 & 14565 & 312 & 1.46 \\
SD & 301 & 94 & 78 & 124 & 144 & 4 & & \\
SD\% & 2.11 & 0.65 & 0.54 & 0.84 & 0.98 & $0.03 \%$ & & \\
\hline
\end{tabular}

The standard deviation of the average of the mean values of all five cycles equals to 4 which is very low compared to the standard deviation of each cycle that ranged from 78 to 301 . Hence, the percentage standard deviation, which is shown in last row of Table (1) for each cycle mean values represent the effect of applying the reproducibility for the group of TLD-600 dosimeters readings.
The values of relative standard deviation (SD\%) ranged from $0.54 \%$ to $2.11 \%$. The value of $0.03 \%$ in the last row of Table (1) gathers the effect of repeatability (five cycles of one dosimeter) and the reproducibility (one cycle for ten dosimeters). It is clear from the results that the element correction factors (ECFs resulted from the repeatability) 
should be applied before calculating the average of different TLD chips.

Figure (3) shows the standard deviation percentage of ten TLDs count before subtracting the background (blue), after subtracting the background (red) and applying element correction factor (ECF) (gray). It was noted that with repeating of the identical procedures (irradiation, annealing, and reading out) additional times, the deviation of the cumulative mean of all chips decrease across all irradiations, as illustrated in Fig. (3).

Reproducibility measurements

a) Calculation of the system variability index (SVI):

The variability of TLDs system (SVI) can be presented by the mean value of the coefficients of variation of every TL dosimeter $\overline{C V}$ expressed in $\%$.

Figure (4) shows the system variability index (SVI) for the mean value of the count, the mean net after subtracting the background and corrected net mean value. The data presented in Fig. (4) are for average of ten dosimeters.

\section{Calculation of reader variability index (RVI)}

The variability of reader variability index (RVI) can be expressed by the coefficients percentage of mean value of the variation of every cycle of readings and this amount provides a long term reader reproducibility.

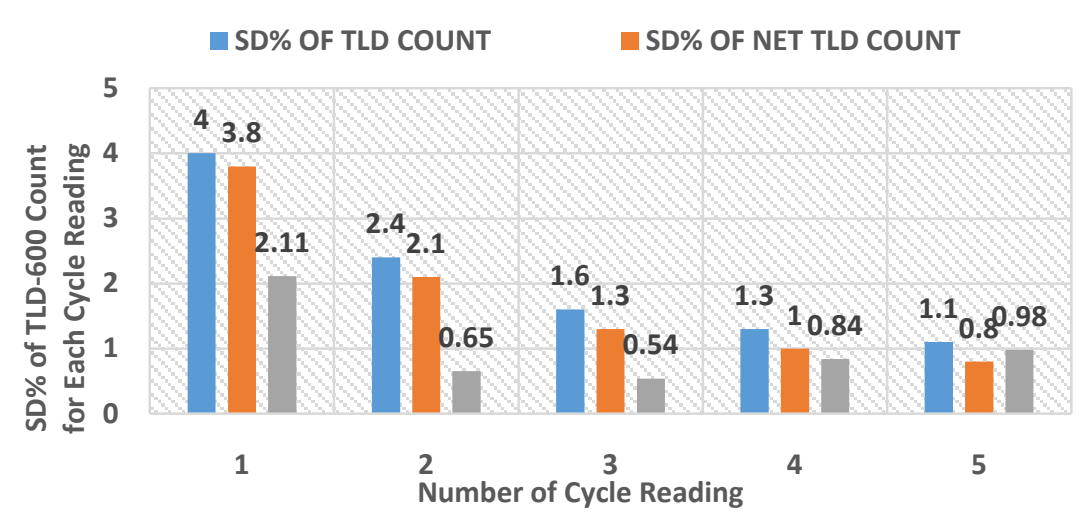

Fig. (3): The change of the mean percent standard deviation of the TLD-600 responses before, after of subtracting the back ground and applying element correction factor (ECF)

Table (2): Calculation of reader variability index (RVI)

\begin{tabular}{|c|c|c|c|c|c|}
\hline \multirow{2}{*}{ TLD-600 count } & \multicolumn{5}{|c|}{ TL reader unit count } \\
\cline { 2 - 6 } & $\mathbf{1}^{\text {st }}$ & $\mathbf{2}^{\text {nd }}$ & $\mathbf{3}^{\text {rd }}$ & $\mathbf{4}^{\text {th }}$ & $\mathbf{5}^{\text {th }}$ \\
\hline mean value of the count & 14497 & 14760 & 14932 & 14994 & 15024 \\
mean net & 14240 & 14487 & 14650 & 14706 & 14730 \\
corrected net mean value & 14237 & 14488 & 14654 & 14710 & 14735 \\
\hline
\end{tabular}

The mean corrected readings for each cycle are shown in last row of Table (2). By determining the coefficients percentage of variation of these results the variability index (RVI) of the TL reader only could be obtained.

Figure (5) shows the TL reader variability index (RVI) for the mean value of the count, the mean net after subtracting the background and corrected net mean value. The data shown in Fig. (5) represents average of 5 cycles as shown in Table
(2). The values presented in Figs. (4 and 5) are nearly the same.

Calculation of the variability index of the detectors (DVI)

From the obtained result for the system variability index (SVI) and TL reader variability index (RVI), the detector variability index (DVI) could be obtained using the formula 4.

Figure (6) shows the values of (SVI), (RVI) and (DVI) for the mean value of the count, the mean net after subtracting the background and corrected 
net mean value. From Fig. (6) it is clear that the applying of the element correction factor dramatically reduces the percentage variation for the detector.
Relative standard deviation versus dose

The variation of the coefficient of variation $S(D)$ of the TL readings at different doses $\mathrm{D}$ can express the reproducibility of a TLD system.

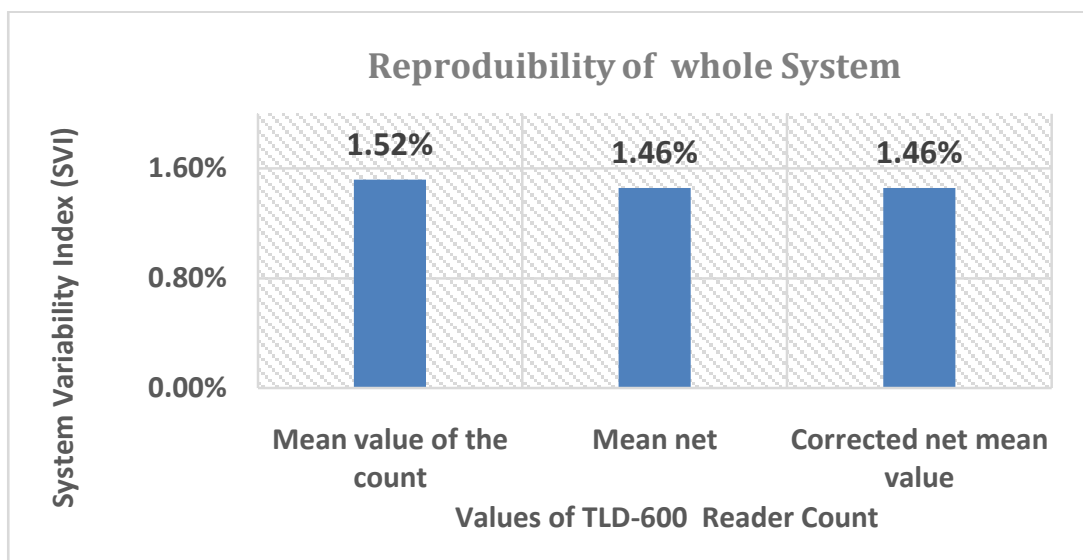

Fig. (4): The change of the mean percent standard deviation of the TLD-600 response before and after of subtracting the back ground and applying element correction factor (ECF)

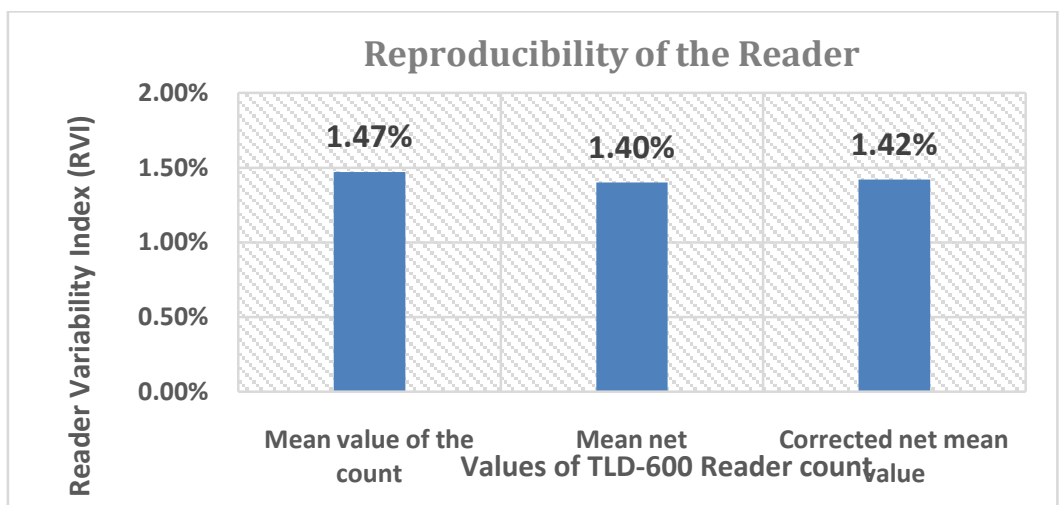

Fig. (5): The change of reader variability index of the TLD-600 response before and after of subtracting the back ground and applying element correction factor (ECF)

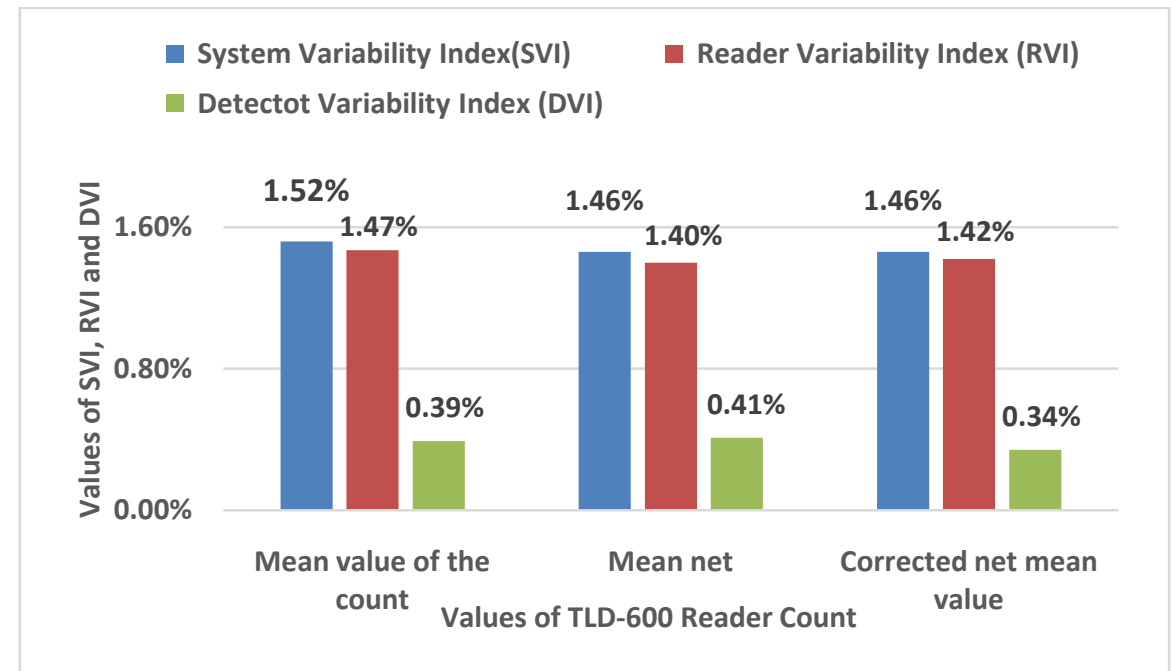

Fig. (6): The change of the SVI, RVI, and DVI values of the TLD-600 response before and after of subtracting the back ground and applying element correction factor (ECF) 
Table (3): Experiential relative of coefficients of variation before and after subtracting of zero dose and correction TL readings

\begin{tabular}{|c|c|c|c|c|c|c|c|c|c|c|c|c|c|}
\hline Dose (mGy) & 0.0038 & 0.019 & 0.0454 & 0.076 & 0.189 & 0.435 & 3.89 & 19.47 & 38.94 & 43.4 & 86.9 & 216.9 & 434 \\
\hline $\begin{array}{c}\text { Experimental } \\
\text { SD\% }\end{array}$ & 18.9 & 14.4 & 13.9 & 11.1 & 12.3 & 11.3 & 4.2 & 2.7 & 2.6 & 2.2 & 2.4 & 2.4 & 2.4 \\
\hline $\begin{array}{c}\text { Experimental } \\
\text { SD\% after } \\
\text { subtracting } \\
\text { zero dose }\end{array}$ & 18.7 & 12.4 & 10.8 & 9.7 & 10.0 & 7.7 & 4.1 & 2.6 & 2.6 & 2.2 & 2.4 & 2.4 & 2.4 \\
\hline $\begin{array}{c}\text { Experimental } \\
\text { SD\% after } \\
\text { correction }\end{array}$ & 16.9 & 10.2 & 9.7 & 8.9 & 7.8 & 6.8 & 3.4 & 1.7 & 1.1 & 1.1 & 1.9 & 0.7 & 0.85 \\
\hline
\end{tabular}

The rows 2, 3 and 4 of Table (3) show the experiential relative of coefficients of variation S(D) at every dose D before and after subtracting of zero and correction TL readings. The coefficients A, B such that the theoretical plot from formula (1) fit the experimental data. From the $2^{\text {nd }}$ row of Table (3) it appears that the coefficient of variation becomes constant at large doses and has a value of $1.4 \%$ and this result matches with formula (1) which referred to the same result.

Figure (7) shows $\mathrm{S}(\mathrm{D}) 2-\mathrm{B} 2$ against $1 / \mathrm{D}^{2} \mathrm{TL}$ readings. When the quantities $S(D) 2-B 2$ against $1 / D^{2}$ were plotted, a linear relation was found with a slop equals to $\mathrm{A} 2=4130.7$ and $\mathrm{B}=1.6$ and this shows that the experimental and the theoretical data are in a reasonable agreement.

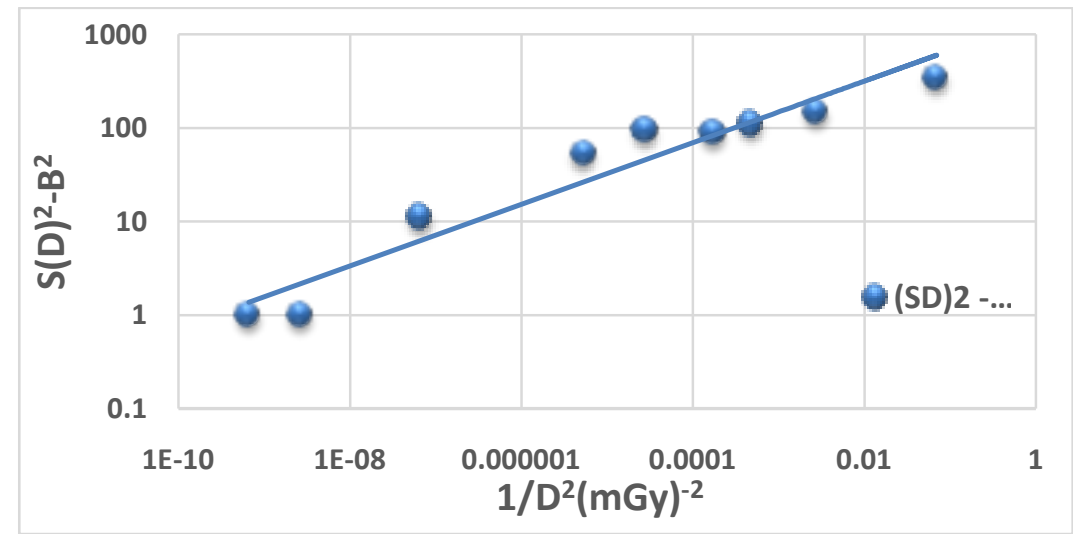

Fig. (7): $S(D)^{2}-B^{2}$ against $1 / D^{2}$.

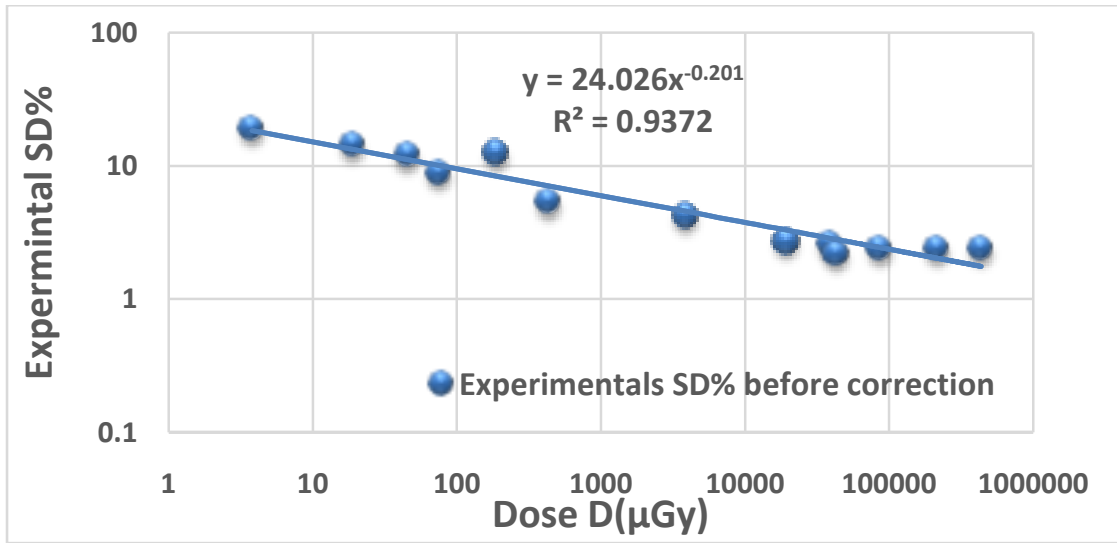

Fig. (8 a): The experimental relative coefficient factor of variation $S(D)$ for 10 TLD-600 dosimeters as a function of dose $D$ 


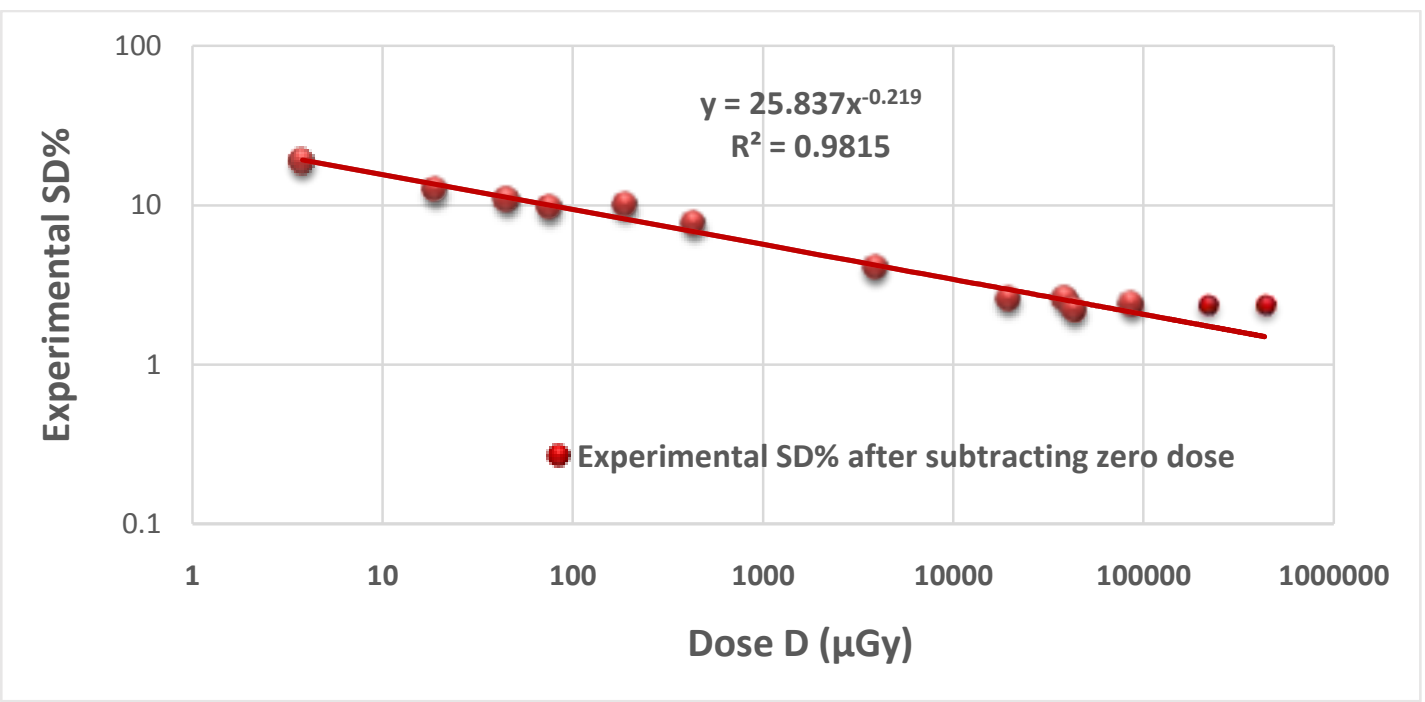

Fig. (8 b): The experimental relative coefficient factor of variation $S(D)$ after subtracting zero dose for 10 TLD-600 dosimeters as a function of dose $D$

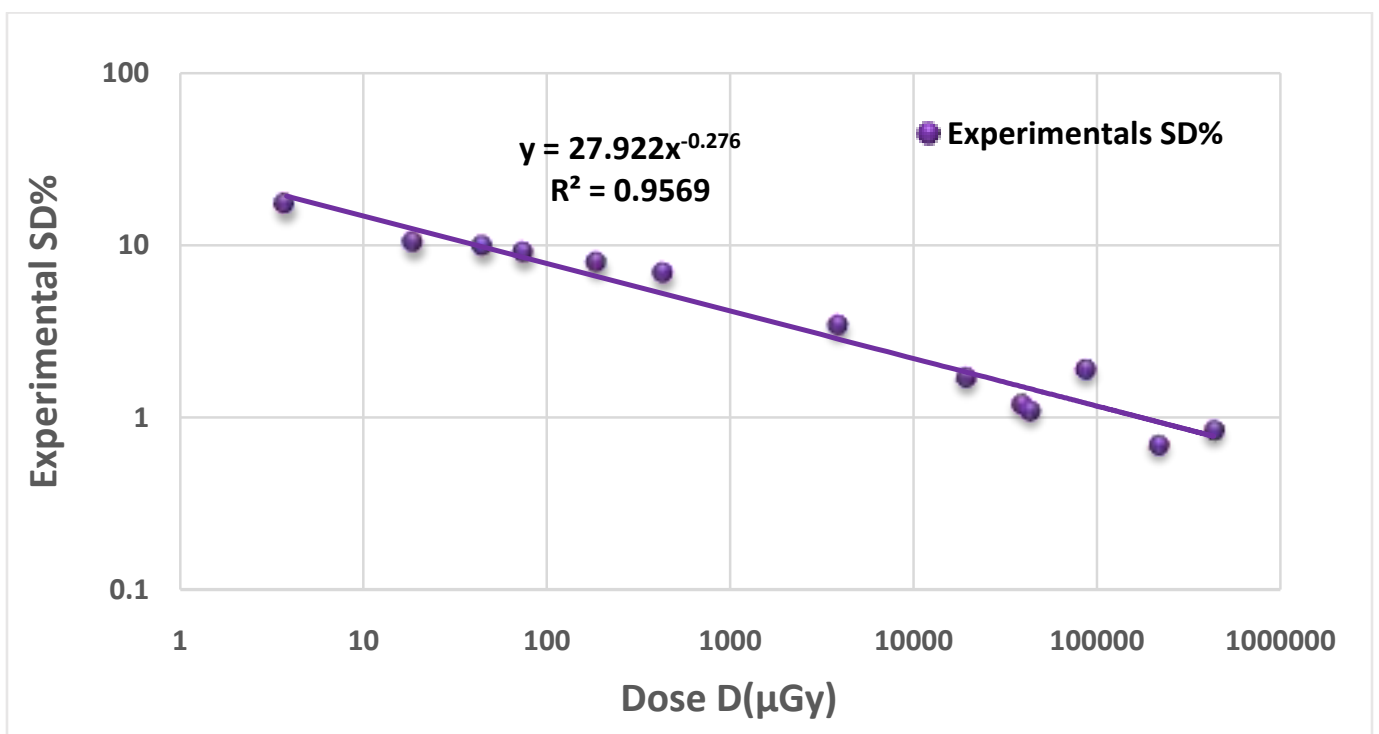

Fig. $(8 \mathrm{c})$ : The experimental relative coefficient factor of variation $S(D)$ after correction for 10 TLD-600 dosimeters as a function of dose $\mathrm{D}$

Figures $(8 \mathrm{a}, 8 \mathrm{~b}$ and $8 \mathrm{c})$ show the experimental relative coefficient factor of variation $S(D)$ for 10 TLD-600 dosimeters as a function of dose D before (blue points), after subtracting zero dose (red point) and correction (violet points) of TL readings. From the results it can be noted that at low doses, the value of relative coefficient of variation becomes very large while at high doses it becomes basically constant and of the order of a few percentage. The value of the constant $S(D)$ is obtained depending on the specifications of the used dosimeters, besides the specifications of the individual TL reader used in the experiment.

\section{Conclusion}

This investigation denotes that more correction will be obtained by calculating the error due to non-subtracting of the zero-dose reading from the delivered dose, which decreases the coefficient of variation as a percentage of the mean of set from the range of $2.4 \%-18 \%$ to only $0.7 \%-16.9 \%$.

After one calibration, the precision of sample measured standard deviation is approximately 
$2.1 \%$. This value was reduced to $0.58 \%$ after three calibrations.

The results indicate that the values of variability index of the system (SVI), the reader (RVI) and the detector (DVI) are $1.46 \%, 1.40 \%$ and $0.34 \%$ respectively and it is apparent that the best repeatability and reproducibility are going to be obtained by using the individual sensitivity factors associated to each dosimeter rather than by carefully selecting the individual dosimeters.

From the results, it can be noted that at low doses, the value of relative coefficient of variation becomes very large while at high doses, it becomes basically constant and of the order of a few percentage.

It is necessary to note that the results which are obtained in this study, may not apply as well to other kinds of thermoluminescent detectors. However, a comparable error analysis could be performed with other different detectors.

\section{References}

1-Sadeghi M, Sina S, Faghihi R.“Investigation of Lif, $\mathrm{Mg}$ and $\mathrm{Ti}$ (TLD-100) Reproducibility." J Biomed Phys Eng. 2015 Dec 1;5(4):217-22. 2015, Dec.

2- Abd El-Hafez A.I. and Maghraby, A. "Impacts of Pre-Irradiation Background Signal On the PostIrradiation Glow Curves of Thermoluminescence Dosimeters" Applied Radiation and Isotopes" 69, (2011), 1533-1539.

3-Abd El-Hafez A.I., Maghraby A. and El-Faramawy N. A. "In Defense Of Thermoluminescence Dosimeter Zero Dose Readouts" Journal of American Science, 2011;7(3) 796-803.

4-Abd El-Hafez A.I., El-Faramawy N., Al-Shareff J.F. and El-Fiki S. "On the Impact of the Pre-Irradiation TLDs Readout on Their Post-Irradiation Glow Curves" International Journal of Physics and Research, Volume 3, Issue 2 (2013) 63-76.

5-Karsch L, Beyreuther E, Burris-Mog T, Kraft S, Richter C, Zeil K, et al. "Dose rate dependence for different dosimeters and detectors: TLD, OSL, EBT films, and diamond detectors." Med Phys. 2012;39:2447-55. doi.org/10.1118/1.3700400. PubMed PMID: 22559615.
6-Nunn AA, Davis SD, Micka JA, DeWerd LA. "LiF:Mg,Ti TLD response as a function of photon energy for moderately filtered $\mathrm{x}$-ray spectra in the range of $20-250 \mathrm{kVp}$ relative to 60Co." Med Phys. 2008;35:1859-69. doi.org/10.1118/1.2898137. PubMed PMID: 18561661.

7-Stadtmann H, Hranitzky C, Brasik N. "Study of real time temperature profiles in routine TLD read out-influences of detector thickness and heating rate on glow curve shape." Radiat Prot Dosimetry. 2006;119:310-3. doi.org/10.1093/ rpd/NCI 655. PubMed PMID: 16825249.

8-Shachar BB, Horowitz Y. "Thermoluminescence in annealed and unannealed $\mathrm{LiF}: \mathrm{Mg}$, Ti (TLD100 Harshaw) as a function of glow curve heating rate and using computerised glow curve deconvolution." Journal of Physics D: Applied Physics. 1992;25:694. doi.org/10.1088/0022- 3727/25/4/016.

9-Lee Y, Won Y, Kang K. "A method to minimise the fading effects of LiF:Mg,Ti (TLD-600 and TLD700) using a pre-heat technique. Radiat Prot Dosimetry." 2015;164:449-55. doi.org/10.1093/rpd/ ncu302. PubMed PMID: 25301971.

10- Doremus S, Higgins G. "Pre-Irradiation Fade and Post-Irradiation Fade for LiF: Mg, Ti, TLD-600, and TLD-700, as a Function of Time. Radiat Prot Dosimetry." 1994;54:119-25.

11-P. Plato and J. Miklos, "Production of Element Correction Factors for Thermoluminiscent Dosimeters." Health Phys. 495 (1985) 873. Health Phys. 49 (5) (1985) 873.

12- B. Burkhardt and E. Piesh, "Reproducibility of TLD systems. A comprehensive analysis of experimental results". Nucl. Instr. Meth. 175 (1980) 159.

13-P. Zarand and I. Polgar, "A theoretical study on the relative standard deviation of TLD systems". Nucl. Instr. Meth. 205 (1983) 525-529. Nucl. Instr. Meth. 205 (1983) 525.

14-P. Zarand and I. Polgar, "on the relative standard deviation of TLD systems", Nucl. Instr. Meth. 222 (1984) 567.

15-S. Del Sol Fern'andez, et al.” Thermoluminescent characteristics of $\mathrm{LiF}: \mathrm{Mg}, \mathrm{Cu}, \mathrm{P}$ and CaSO4:Dy for low dose measurement." Applied Physics and Isotopes. Volume 111, May 2016, Pages 50-55. 